\title{
Label-free optical biosensor on a single chip
}

Miguel Holgado, Rafael Casquel, and María-Fe Lagunas

Combining semiconductor technology with photonics, optics, and biochemistry leads to sensors with improved biomedical diagnostic capability.

The healthcare and pharmaceutical sectors continuously demand more powerful analytical and diagnostic tools for the identification of disease, the development of new medicines, and better diagnostic tests. High-capability optical biosensing systems are emerging ${ }^{1}$ as a way to achieve these goals. Based on the combination of optical materials and photonic components, optical sensing offers many advantages. For instance, the technology is non-invasive, immune to electromagnetic interference, and the risk of electrical shocks or explosions is nonexistent. A key enabler for these systems is the development of costeffective miniaturization technology, which permits the efficient integration of mechanical, fluidic, and photonic components.

In typical biosensors, detection of specific pathogens or proteins begins by immobilizing appropriate bioreceptors on the sensing areas of a chip. When analytes are introduced into those areas, only the target biomolecules will be bound to their corresponding biomolecular receptors. This binding process is usually monitored with commercial analytical techniques that require transduction labeling elements, such as fluorescent dyes or radioactive isotopes, to generate a physically readable signal from a recognition event. However, labeling chemistry is expensive and time-consuming. In contrast, recent progress in biosensing systems, in which biomolecules are unlabeled or unmodified (label-free biosensing), is showing promising results. ${ }^{2}$

The sensing transduction signals in optical label-free biosensing platforms are based on small changes in refractive index caused by the immobilization or binding reaction of biomolecules. Planar photonic biosensors have measured biomolecular binding interactions using well-known photonic structures, including surface plasmon resonance, ${ }^{3,4}$ interferometers, ${ }^{5-8}$ resonators, ${ }^{9,10}$ gratings, ${ }^{11,12}$ and photonic crystals. ${ }^{13,14}$ These photonic sensing biochips are attractive because they integrate multiple compact devices on a single chip. But, although they use low cost mature semiconductor technology, planar photonic biochips normally need complex optical coupling systems, such as inverted taper and grating couplers, which make the devices less cost effective.

Our vision is to create a highly sensitive, label-free optical biosensing system that is fully compatible with semiconductor technology and can be fabricated and interrogated at wafer or disposable chip level. Our holistic approach combines biophotonic resonant or interferometric sensing cells ${ }^{15-19}$ and advanced micron and sub-micron spot size high-sensitivity optical interrogation technologies. This approach leads to a new generation of high-value biochips that are scalable at the wafer level without the need for complex coupling systems, thus offering an inexpensive solution for integration and packaging.

Our optical sensing system uses a tightly focused beam (see Figure 1), which allows in situ measurement of micron and submicron size geometries. In our current work, we have already demonstrated the capability to measure extremely small volumes of analytes (on the order of $0.1 \mathrm{fL}$ per hole). ${ }^{15,16}$ This system makes routine screening more cost effective and, because each wafer can integrate hundreds of sensing structures, it is also suitable for performing hundreds of measurements on one or multiple samples with high throughput and on-wafer monitoring. To determine the interactions with receptor biomolecules with more reliability and sensitivity, the system will be tremendously sensitive to refractive index variations: on the order of $10^{-6}$ or even $10^{-7}$ refractive index units (RIUs). This means that direct detection of picomolar or even lower concentrations is feasible. Both observation of the reflectivity profile and magnification of the biophotonic resonant or interferometric sensing cells will facilitate this sensitivity.

Label-free optical biosensors are expected to grow most in importance in the healthcare, biomedical, and biopharmaceutical sectors, since they can provide new analytical tools with reduced size as well as facilitate large-scale screening of a very wide range of samples for many different parameters. Our optical biosensing system, which uses mixed technologies, such as op-

Continued on next page 


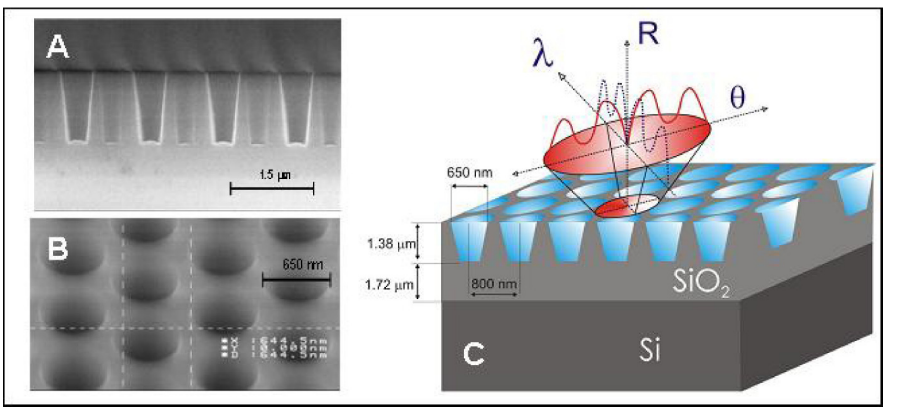

Figure 1. Scanning electron micrographs of an example of an interferometric photonic sensing cell. (A) Cross-section. (B) Top view. (C) A schematic representation of the optical techniques employed to interrogate a biosensing cell. The laser beam is focused on the center of a sub-micron hole into which potential analytes can be introduced. The volume per hole is on the order of femtoliters. Light is characterized by wavelength $(\lambda)$, angle of incidence $(\theta)$, and reflectivity $(R)$.

tics and bio-photonics, at micro-scale integration, is intended to be more cost effective and have more capabilities than those currently available. It will take advantage of semiconductor technology, not only from the point of view of integration, but also in the way we interrogate the biochips. With the simultaneous use of three different techniques, the system will be much more reliable, removing ambiguities during the sensing interrogation process and achieving higher throughput and productivity in comparison with other established analytical techniques. Eventually, these biosensors have the potential to bring the lab not only to the hospital but also to end users.

\section{Author Information}

\section{Miguel Holgado and María-Fe Lagunas \\ Laser Center and}

Escuela Técnica Superior de Ingeniería Industrial, Department of Applied Physics

Technical University of Madrid

Madrid, Spain

Miguel Holgado was an engineer in the laser section of the Spanish Ministry of Defense from 1991 to 1994 and at the Institute of Material Science of Madrid from 1994 to 1997. From 1997 to 2001 he worked at Lucent Technology Microelectronics. From 2001 to 2004 he was the Spanish delegate to the Information Society Technologies Framework Program, and in 2005 became head of R\&D projects at the Nanophotonic Technology Center in Valencia, Spain. Currently he is the team leader in micronano photonics at the Technical University of Madrid Laser Center.

\section{Rafael Casquel}

Laser Center

Technical University of Madrid

Madrid, Spain

\section{References}

1. X. Fan, I. M. White, S. I. Shopova, H. Zhu, J. D. Suter, and Y. Sun, Sensitive optical biosensors for unlabeled targets: a review, Anal. Chim. Acta 620, pp. 8-26, 2008. doi:10.1016/j.aca.2008.05.022

2. A. M. Armani, R. P. Kulkarni, S. E. Fraser, R. C. Flagan, and K. J. Vahala, Labelfree, single-molecule detection with optical microcavities, Science 317, pp. 783-786, 2007. doi:10.1126/science. 1145002

3. R. Ince and R. Narayanaswamy, Analysis of the performance of interferometry, surface plasmon resonance and luminescence as biosensors and chemosensors, review, Anal. Chim. Acta 569 (1-2), pp. 1-20, 2006. doi:10.1016/j.aca.2006.03.058

4. J. Homola, S. Yee, and G. Gauglitz, Surface plasmon resonance sensors: review, Sens. Actuators, B 54 (1-2), pp. 3-15, 1999. doi:10.1016/S0925-4005(98)00321-9

5. B. J. Luff, J. S. Wilkinson, J. Piehler, U. Hollenbach, J. Ingenhoff, and N. Fabricius, Integrated optical Mach-Zehnder biosensor, J. Lightwave Technol. 16 (4), pp. 583-592, 1998.

6. F. Prieto, B. Sepúlveda, A. Calle, A. Llobera, C. Domínguez, A. Abad, A. Montoya, and L. M. Lechuga, An integrated optical interferometric nanodevice based on silicon technology for biosensor applications, J. Nanotechnol. 14, pp. 907-912, 2003. doi:10.1088/0957-4484/14/8/312

7. F. Prieto, B. Sepúlveda, A. Calle, A. Llobera, C. Domínguez, and L. M. Lechuga, Integrated Mach-Zehnder interferometer based on ARROW structures for biosensor applications, Sens. Actuators, B 92, pp. 151-158, 2003. doi:10.1016/S0925-4005(03)00257-

8. G. H. Cross, A. A. Reeves, S. Brand, J. F. Popplewell, L. L. Peel, M. J. Swann, and N. J. Freeman, A new quantitative optical biosensor for protein characterisation, Biosens. Bioelectron. 19 (4), pp. 383-390, 2003. doi:10.1016/S0956-5663(03)00203-3

9. C. A. Barrios, M. J. Bañuls, V. González-Pedro, K. B. Gylfason, B. Sánchez, A. Griol, A. Maquieira, H. Sohlström, M. Holgado, and R. Casquel, Labelfree optical biosensing with slot-waveguides, Opt. Lett. 33 (7), pp. 708-710, 2008. doi:10.1364/OL.33.000708

10. A. Ksendzov and Y. Lin, Integrated optics ring-resonator sensors for protein detection, Opt. Lett. 30 (24), pp. 3344-3346, 2005. doi:10.1364/OL.30.003344

11. J. Vörös, J. J. Ramsden, G. Csúcs, I. Szendró, S. M. De Paul, M. Textor, and N. D. Spencer, Optical grating coupler biosensors, Biomaterials 23 (17), pp. 3699-3710, 2002. doi:10.1016/S0142-9612(02)00103-5

12. M. Wiki and R. E. Kunz, Wavelength-interrogated optical sensor for biomedical applications, Opt. Lett. 25 (7), pp. 463-465, 2000. doi:10.1364/OL.25.000463

13. E. Chow, A. Grot, L. W. M. Mirkarimi, M. Sigalas, and G. Girolami, Ultracompact biochemical sensor built with two dimensional photonic crystal microcavity, Opt. Lett. 29 (10), pp. 1093-1095, 2004. doi:10.1364/OL.29.001093

14. P. Domachuck, H. Nguyen, H. C. Eggleton, B. J. Straub, and M. Gu, Microfluidic tunable photonic band-gap device, Appl. Phys. Lett. 84, pp. 1838-1840, 2004. doi:10.1063/1.1667592

15. M. Holgado, R. Casquel, C. Molpeceres, M. Morales, and J. L. Ocaña, Simultaneous reflectivity, ellipsometry and spectrometry measurements in submicron structures for liquid sensing, Sens. Lett. 6 (4), pp. 564-569, 2008. doi:10.1166/sl.2008.430

16. M. Holgado, R. Casquel, B. Sánchez, C. Molpeceres, M. Morales, and J. L. Ocaña, Optical characterization of extremely small volumes of liquid in sub-microholes by simultaneous reflectivity, ellipsometry and spectrometry, Opt. Express 15 (20), pp. 13318-13329, 2007. doi:10.1364/OE.15.013318

17. M. Holgado, R. Casquel, María-Fe Laguna, M. Morales, C. Molpeceres, and J. L. Ocaña, Sub-micro holes sensing cells analyzed by Fourier transform IR-visible spectrometry for biochemical sensing, Proc. Eurosensors, pp. 668-671, 2008. ISBN 978-300-025217-4

18. R. Casquel, M. Holgado, A. Lavín, C. A. Barrios, C. Molpeceres, M. Morales, and J. L. Ocaña, Vertical resonant microcativites based on pillars analyzed by beam profile ellipsometry and reflectometry, Proc. Eurosensors, pp. 1577-1580, 2008. ISBN 978-300-025217-4 
19. M. Holgado and R. Casquel, Micro-nano photonic biosensors scalable at the wafer level, 2009. Paper accepted at OPTO: Integrated Optoelectronic Devices, SPIE Photonics West in San Jose, California, January 24-29, 2009. 